\title{
Usefulness of Bioelectrical Impedance Analysis as a Guidance of Fluid Management in Critically III Patients after Major Abdomen Surgery; a Single Center, Prospective Cohort Study
}

\author{
Yoon Ji Chung, M.D. ${ }^{1}$, Eun Young Kim, M.D., Ph.D. ${ }^{2}$ \\ ${ }^{1}$ Department of Surgery, Seoul St. Mary's Hospital, ${ }^{2}$ Division of Trauma and Surgical Critical Care, Department of Surgery, Seoul St. \\ Mary's Hospital, College of Medicine, The Catholic University of Korea, Seoul, Korea
}

Purpose: We evaluated the patterns and changes in bioelectrical impedance analysis parameters of patient who underwent abdominal surgery throughout the early period in surgical intensive care unit stay.

Materials and Methods: From May 2019 to April 2020, patients admitted to surgical intensive care unit for more than 48 hours after surgery were enrolled. Body composition and volume status of patients were measured prospectively using portable bioelectrical impedance analysis device every morning for three days from the day of intensive care unit admission. Overhydration was defined as the case where the value of extracellular water ratio is above 0.390 , and the participants were daily classified into overhydration or normohydration group. Relationship between daily volume status measured by bioelectrical impedance analysis and outcomes was assessed.

Results: 107 patients who underwent abdominal surgery and 26 patients who underwent endovascular surgery were reviewed as control group. During the first postoperative 48 hours, most of them showed overhydration status, while the rate of overhydaration was significantly lower in the control group. Overhydration status on day 3 was significant predictors of postoperative morbidities (OR 5.709, 95\% CI 2.199 14.819, P<0.001) and in-hospital mortality (OR 4.244, 95\% CI 1.398 12.883, P<0.001).

Conclusion: Overhydration status by extracellular water ratio on postoperative day 3 needs careful monitoring and appropriate interventions to improve the postoperative morbidities and in-hospital mortality. Bioelectrical impedance analysis could be a simple, easy and useful tool to monitor the volume status of patients who requiring intensive fluid resuscitation after abdominal surgery.

Keywords: Bioelectrical impedance analysis, Intensive care unit, Morbidity, Mortality, Volume status

\section{INTRODUCTION}

Critically ill patients underwent abdominal major surgery commonly have a wide range of organ injuries, damage of vascular structure or abdominal wall, or extensive tissue removal through surgery that inevitably leads to severe systemic inflammatory reactions and physiological stress. Thus, the fluid resuscitation and close monitoring in intensive care unit (ICU) is usually needed in order to correct hemodynamic instability, maintain proper organ perfusion and also compensate for severe fluid depletion mainly due to fluid loss during surgery. However, no precise guidance for optimal fluid

Received October 14, 2020; Revised November 12, 2020; Accepted November 26, 2020

Corresponding author: Eun Young Kim

E-mail freesshs@naver.com (iD) ORCID https://orcid.org/0000-0003-4408-4165

(1) (8) This is an Open Access article distributed under the terms of the Creative Commons Attribution Non-Commercial License (http://creativecommons.org/licenses/ (c) by-nc/4.0), which permits unrestricted non-commercial use, distribution, and reproduction in any medium, provided the original work is properly cited. 
management after major abdominal operations has been found. For assessment of the adequacy in fluid therapy, various monitoring tools such as serum lactate, venous saturation or daily fluid balance have been used in clinical settings but they have limitations such as ease of use or accuracy of results.[1-3] As a result, most surgeons have still performed the fluid therapy after abdominal surgery based on clinician's judgment or experience in absence of the evidence-based guidelines.

Bio-Impedance analysis (BIA) is a device that measures the impedance such as resistance value caused by the difference in the electrical conductivity of each type of biological tissue such as muscle and fat. The electrical conductivity in tissue is proportional to the amount of electrolyte or water, that allows BIA to measure the amount of water quantitatively in and out of cells as well as muscle mass and fat in body and to also estimate the status of body composition. In fact, many studies have previously reported that BIA would be feasible for assessing the volume status and predicting the prognosis of critical ill patients.[1,2,4] However, there has been few studies to evaluate the adequacy of volume replacement therapy and clinical usefulness in patients after major abdomen surgery using BIA.

Herein, we assessed the relationship between changes in various BIA parameter values and response to fluid therapy of critically ill patient during the intensive care unit (ICU) stay after major abdominal surgery. The relationship of BIA parameters indicating volume status and postoperative outcomes was also evaluated, and the significant BIA parameters for predicting of prognosis was identified.

\section{MATERIALS AND METHODS}

\section{Participants}

This is a prospective observational study that was performed in our 22-bed surgical ICU of a single tertiary academic teaching hospital. From May 2019 to April 2020, all patients aged over 18 admitted to the SICU after abdomen surgery under general anesthesia were eligible for inclusion regardless of type of surgical procedure or clinical department. Patients who admitted surgical ICU after endovascular surgery were also included and analyzed as control group. If patients who met any one of those findings same as follows, they were excluded from study enrollment; 1 ) length of ICU stay within 48 hours, 2) who underwent any other surgeries unless abdominal or endovascular surgery, 3) any presence of prosthetic medical devices such as pacemakers or metallic intravascular device, 4) underwent extracorporeal membrane oxygenation treatment or diuretic treatment, 5) had bone fixation implants or limb amputation, 6) undergoing routine hemodialysis or receiving continuous renal replacement therapy (CRRT), 7) gravidity, 8) readmission within 48 hours after discharge from the ICU, 9) admitted SICU only for medical causes without operation, or 10) obtained the informed con- sent for do-not-resuscitation or inability to provide informed consent.[1-3] Patients who expired within 72 hours after surgery were also excluded for analysis. The current study was approved and carefully monitored by our Institutional Review Board (No. IRB; KC19RESI0584).

\section{BIA measurement and conventional volume assessment}

In the current study, the body composition status was assessed using a commercial portable BIA device using 50$\mathrm{kHz}$ alternating current. (InBody S10 ${ }^{\circledR}$, InBody Corp., Seoul, Korea).[1] This InBody S10 analyzer used adhesive-type or touch-type electrodes attached to four limbs and designed for patients older than 3 years.

After patients were postoperatively admitted to the surgical $\mathrm{ICU}$, the BIA measurements were performed daily for three days every morning (from Day 1 to Day 3 ). It was performed while patients were lying posture on the bed (Fig. 1). For touch type electrodes, 6 electrodes were used; one on the thumb of each hand, one on each middle finger and one on each inner ankle. To prevent any measurement error due to inappropriate postures or electrodes attachments, BIA measurements were performed by one well-trained physician [1] and the time required for BIA measurement is about only two minutes for each participant. For each participant, the following parameters were obtained; extracellular water (ECW), intracellular water (ICW), total body water (TBW) in liters (L), ECW ratio, skeletal muscle mass, whole-body and segmental phase angle.[1,2] ECW ratio was defined as the ratio of ECW to TBW, as ECW/TBW. The phase angle was defined as the physiological index of cell membrane integrity and vitality that it could estimate the quality and quantity of the soft tissue. The phase angle for each body segment and whole body are calculated by the formula same as follows.

$$
\varnothing=\arctan \left(57.296 \times X_{c} / R\right)
$$

( $\varnothing$ is phase angle; $X_{c}$ is reactance; $R$ is the resistance)

The phase angle is usually regarded to present the cellularity or the integrity of cellular membrane, and also represents the direct ratio between resistance and reactance that could imply that functional cell membrane alterations.[4,5]

As a means to evaluate conventional static parameters of volume status, the daily fluid balance was recorded and reviewed. It was defined as the difference between daily total fluid intake including both intravenous and enteral fluid administered, blood products or medications and total output including all losses through urinary, gastrointestinal or other drainage tubes. The fluid balance represents the total intake and output of the previous day, which was measured collectively at 6 a.m of measurement day. That is an intake and output from 6 a.m. the day before to 6 a.m. on the day of the measurement. For instance, the fluid balance of day 3 is the accumulated intake and output from 6 a.m. of day 2 to 6 a.m. of day 3 . Urine output was hourly measured through 


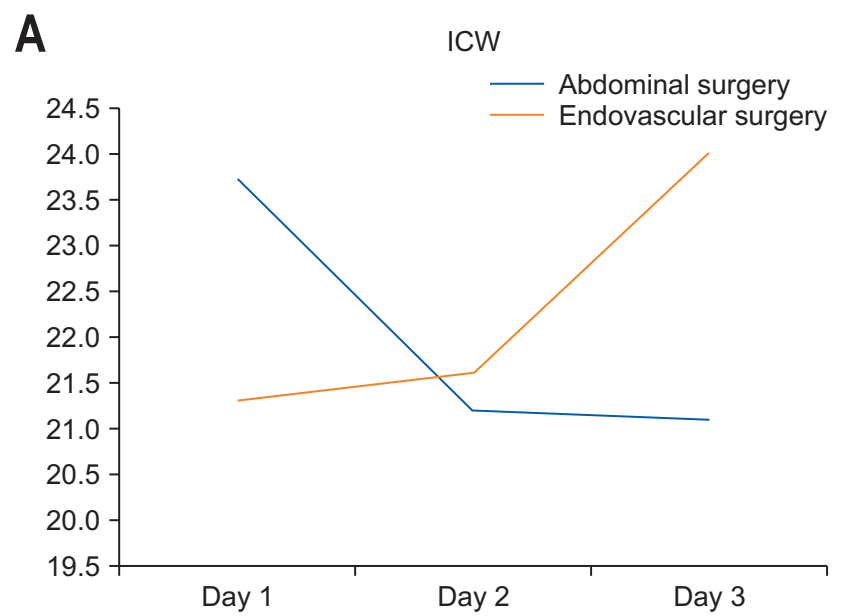

B
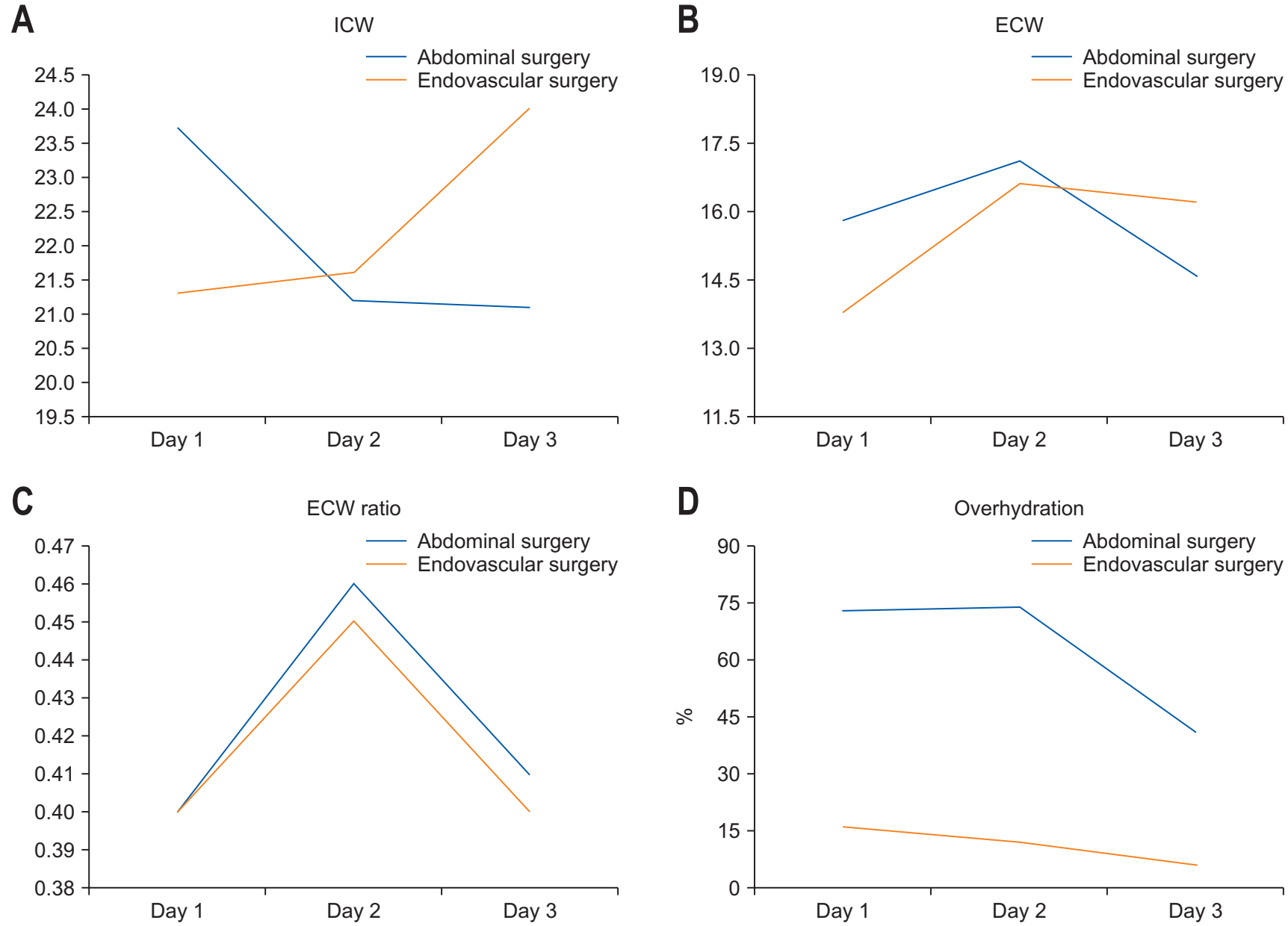

D

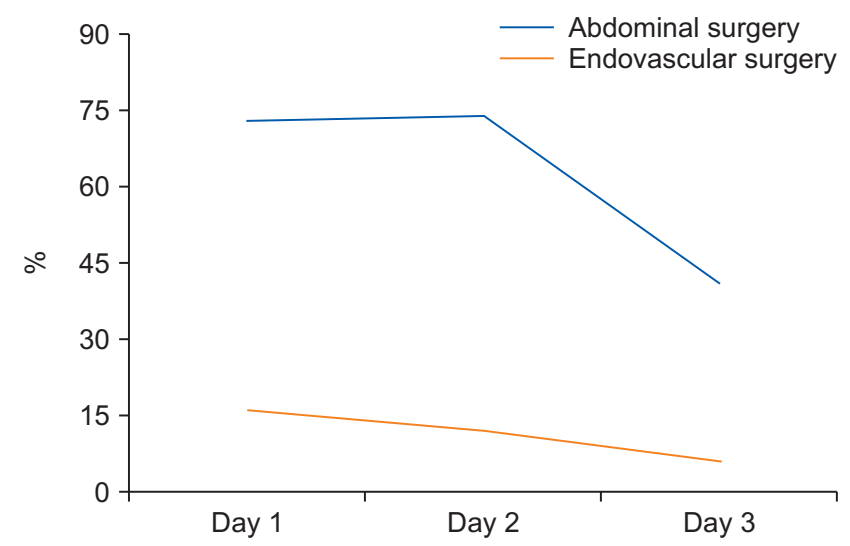

Fig. 1. Daily variations of each volumetric variable of BIA during first 3 days after surgery in the patients underwent abdominal surgery and those in the control group who underwent endovascular surgery. (A) Daily variations of ICW in two groups with the absolute value, (B) Daily variations of ECW in two groups with the absolute value, (C) Daily variations of ECW ratio defined as the ratio of ECW to TBW (ECW/TBW) in two groups, (D) Percentage of the patients in overhydration status been ECW ration above 0.390 in two groups.

foley catheter and the daily fluid balance was calculated and recorded on a specifically designed document by the trained nurses every 24 hours.

\section{Other data recruitment and clinical outcome}

In current study, the clinical data and outcomes were prospectively collected and recorded for enrolled participants. From the electronic medical charts and operative records, we reviewed data such as follows; the demographic data including age, gender, body mass index or the presence of underlying disease, the disease characteristics and severity index using Acute Physiology and Chronic Health Evaluation II (APACHE II) and the sequential organ failure assessment (SOFA) score at ICU admission, and the presence of shock. For the surgical profiles, the name of surgery, operation time and the estimated blood loss were reviewed. During the ICU stay, postoperative morbidities which defined as grade 3 or more according to the Clavien-Dindo classification [6] were recorded in this study. Therefore, the performing of any specialized medical intervention to achieve homeostasis and to preserve organ function such as renal replacement therapy such as hemodialysis or CRRT, the mechanical ventilation support or extracorporeal membrane oxygenation were also recorded. Any clinical complications were closely monitored such as development of pneumonia, pulmonary edema or acute kidney injury (AKI). The ICU or hospital mortality were defined as the deaths occurring during the ICU stay or the hospitalization period.

\section{Statistical analysis}

In the current study, the continuous data were presented as the mean \pm standard deviation (SD) and the overall differences were tested by Student's t-test or ANOVA. The categorical variables were presented as proportions, and were calculated using the chi-square $\left(\chi^{2}\right)$ test or Fisher's exact test. The Pvalue of less than 0.05 was considered statistically significant. 
According to the result of previous study, [7-9] the ECW ratio of more than 0.390 was defined as the overhydration group, and patients were compared for each day dividing by the overhydration group $(\mathrm{OH})$ and the normohydration group $(\mathrm{NH})$. We examined the correlation between the BIA parameters estimated daily for postoperative three days from ICU admission and the other clinical indicators used to reflect volume status such as the value of daily fluid balance. The difference in the clinical outcome was analyzed according to the volume status classified by the ECW ratio on Days 1 and 3, respectively. For multivariate analysis, the logistic regression analysis was conducted for development of the postoperative complications or in-hospital mortality. All the statistical analyses were conducted using SPSS statistical package software for Windows (version 21.0; SPSS Inc., Chicago, IL, USA).

\section{RESULTS}

A total of 107 patients who admitted at SICU of our institution after abdomen operation from May 2019 to April 2020 were analyzed. Patient demographics and clinical outcomes are shown in Table 1. Mean age of patients was $66.9 \pm 15.5$ (range, 19 97), and mean APACHE II score and SOFA score measured at ICU admission were 13.5 and 3.4, respectively. Mean operative time was $201.4 \pm 125$ minutes and the estimated blood loss during operation was $406 \pm 930.6 \mathrm{~mL}$. Mean length of ICU stay and hospital stay were 5.1 days and 22.5 days, respectively. Patients diagnosed as shock status accounted for $16.8 \%$. The ICU mortality was $4.7 \%$ and inhospital mortality was $19.6 \%$ (Table 1 ).

The daily value of BIA parameters for 3 days was shown in Fig. 1 that demonstrated the changes of value in patient underwent abdominal surgery and the changes in the control group who underwent endovascular surgery (26 patients). The number of patients with overhydration status was increased to Day 2 (74 patients, $69.2 \%$ ), followed by a decline to $38.3 \%$ on Day 3 while the control group had a small percentage of overhydrated patients and less daily changes in fluid status. The comparable analyses between the $\mathrm{OH}$ and the $\mathrm{NH}$ on Day 1 and 3 are shown in Table 2 and Fig. 2. There was no difference in clinical outcome between the $\mathrm{OH}$ and $\mathrm{NH}$ groups on Day 1, but the postoperative morbidities and in-hospital mortality in the $\mathrm{OH}$ group were significantly higher than in the $\mathrm{NH}$ group on Day 3. The length of ICU stay and hospital stay also increased significantly in the $\mathrm{OH}$ group on Day 3 than $\mathrm{NH}$ group, while there was no difference between the $\mathrm{OH}$ and $\mathrm{NH}$ group on Day 1 .

The results of the multivariate analysis to identify predisposing factors of postoperative morbidity occurrence and inhospital mortality were presented in Table 3A and Table 3B. Postoperative morbidity (odds ratio (OR): $5.709,95 \% \mathrm{Cl}$ : 2.199 14.819, $\mathrm{P}<0.001$ ) and in-hospital mortality (OR: 4.244, 95\% Cl: $1.398 \sim 12.883, \mathrm{P}=0.045)$ were significantly more likely to occur in patient with overhydration status in Day 3.

\section{DISCUSSION}

This study shows that the overhydration level of most ICU patients after abdomen surgery usually reaches its peak on Day 2 of the ICU care and then the volume status tends to be decreased. The multivariate analysis shows that the ECW ratio on Day 3 among various BIA factors is the most valid factor as predictor of postoperative morbidities and in-hospital mortality.

In the cases of postoperative patients after having major surgery, the fluid imbalance is often observed right after the

Table 1. Demographic characteristics and clinical outcomes of study population

\begin{tabular}{|c|c|}
\hline Variables & $\mathrm{N}=107(\%)$ \\
\hline \multicolumn{2}{|l|}{ Demographic characteristics } \\
\hline Age, years & $66.9 \pm 15.5$ \\
\hline \multicolumn{2}{|l|}{ Gender } \\
\hline Male/Female & $62 / 45(57.9 / 42.1)$ \\
\hline APACHE II score at ICU admission & $13.5 \pm 6.9$ \\
\hline SOFA score at ICU admission & $3.4 \pm 3.3$ \\
\hline Hypertension & $51(47.7)$ \\
\hline Diabetes & $33(30.8)$ \\
\hline COPD & $6(5.6)$ \\
\hline Heart failure & $5(4.7)$ \\
\hline End-stage liver disease & $4(3.7)$ \\
\hline End-stage renal disease & $7(6.5)$ \\
\hline Malignancy & $53(49.5)$ \\
\hline \multicolumn{2}{|l|}{ Operative profiles } \\
\hline Minimal invasive surgery & $36(33.6)$ \\
\hline Operative time, $\min$ & $201.4 \pm 125$ \\
\hline Estimated blood loss, mL & $406 \pm 930.6$ \\
\hline \multicolumn{2}{|l|}{ Type of surgery } \\
\hline Upper GI surgery & $23(21.5)$ \\
\hline Lower GI surgery & $21(19.6)$ \\
\hline Hepato-biliary-pancreas surgery & $34(31.8)$ \\
\hline Gynecologic or obstetric surgery & $7(6.5)$ \\
\hline Trauma & $10(9.3)$ \\
\hline Miscellaneous & $12(11.2)$ \\
\hline \multicolumn{2}{|l|}{ Clinical outcomes } \\
\hline Presence of shock & $18(16.8)$ \\
\hline Mechanical ventilation & $16(15)$ \\
\hline CRRT or HD & $5(4.7)$ \\
\hline ECMO & $1(0.9)$ \\
\hline Length of ICU stay, day & $5.1 \pm 4.7$ \\
\hline Length of hospital stay, day & $22.5 \pm 16.6$ \\
\hline ICU mortality & $5(4.7)$ \\
\hline In-hospital mortality & $21(19.6)$ \\
\hline
\end{tabular}


Table 2. Comparative analysis of volumetric variables and clinical outcomes according to the status of hydration* measured by BIA at postoperative day 1

\begin{tabular}{|c|c|c|c|c|c|c|}
\hline \multirow[b]{2}{*}{ Variables } & \multicolumn{3}{|c|}{ Day 1} & \multicolumn{3}{|c|}{ Day 3} \\
\hline & $\begin{array}{c}\mathrm{OH}^{*} \\
(\mathrm{n}=73,68.2 \%)\end{array}$ & $\begin{array}{c}\text { NH } \\
(n=34,31.8 \%)\end{array}$ & P-value & $\begin{array}{c}\mathrm{OH}^{*} \\
(\mathrm{n}=41,38.3 \%)\end{array}$ & $\begin{array}{c}\text { NH } \\
(n=66,61.7 \%)\end{array}$ & P-value \\
\hline Daily fluid balance at day $1, \mathrm{~mL}$ & $748.6 \pm 999.8$ & $467.3 \pm 1156.6$ & 0.202 & & & \\
\hline Daily fluid balance at day $3, \mathrm{~mL}$ & & & & $412.1 \pm 1469.4$ & $256.6 \pm 1295.4$ & 0.675 \\
\hline Age, years & $69 \pm 134$ & $63 \pm 18$ & 0.069 & $69.3 \pm 12.7$ & $65.5 \pm 16.1$ & 0.200 \\
\hline SOFA score at ICU admission & $3.8 \pm 2.9$ & $2.5 \pm 3.8$ & 0.057 & $3.9 \pm 3.2$ & $3.1 \pm 3.3$ & 0.231 \\
\hline Operative time, $\min$ & $214.5 \pm 136.6$ & $171.6 \pm 87.8$ & 0.057 & $216.1 \pm 135.4$ & $192 \pm 118$ & 0.337 \\
\hline Estimated blood loss, mL & $365.3 \pm 737.7$ & $497.7 \pm 1268.1$ & 0.507 & $464.3 \pm 907.7$ & $368.2 \pm 950.8$ & 0.610 \\
\hline Postoperative morbidities** & $42(57.5)$ & $13(38.2)$ & 0.096 & $32(78)$ & $23(34.8)$ & $<0.001$ \\
\hline Length of ICU stay, day & $5.1 \pm 4.7$ & $5.2 \pm 4.8$ & 0.957 & $7.6 \pm 6.6$ & $3.6 \pm 1.9$ & $<0.001$ \\
\hline Length of hospital stay, day & $24.2 \pm 18$ & $18.9 \pm 12.5$ & 0.125 & $27.1 \pm 18.5$ & $19.7 \pm 14.7$ & 0.024 \\
\hline ICU mortality & $3(4.1)$ & $2(5.9)$ & 1.000 & $2(4.9)$ & $3(4.5)$ & 1.000 \\
\hline In-hospital mortality & $14(19.2)$ & $7(20.6)$ & 1.000 & $14(34.1)$ & $7(10.6)$ & 0.005 \\
\hline
\end{tabular}

*Overhydration is defined as the case where the value of ECW ratio (ECW/TBW) is above 0.390.[7-9]

**Postoperative morbidities were defined as the occurrence of morbidities as grade 3 or more according to the Clavien-Dindo classification.[6]

Length of ICU stay
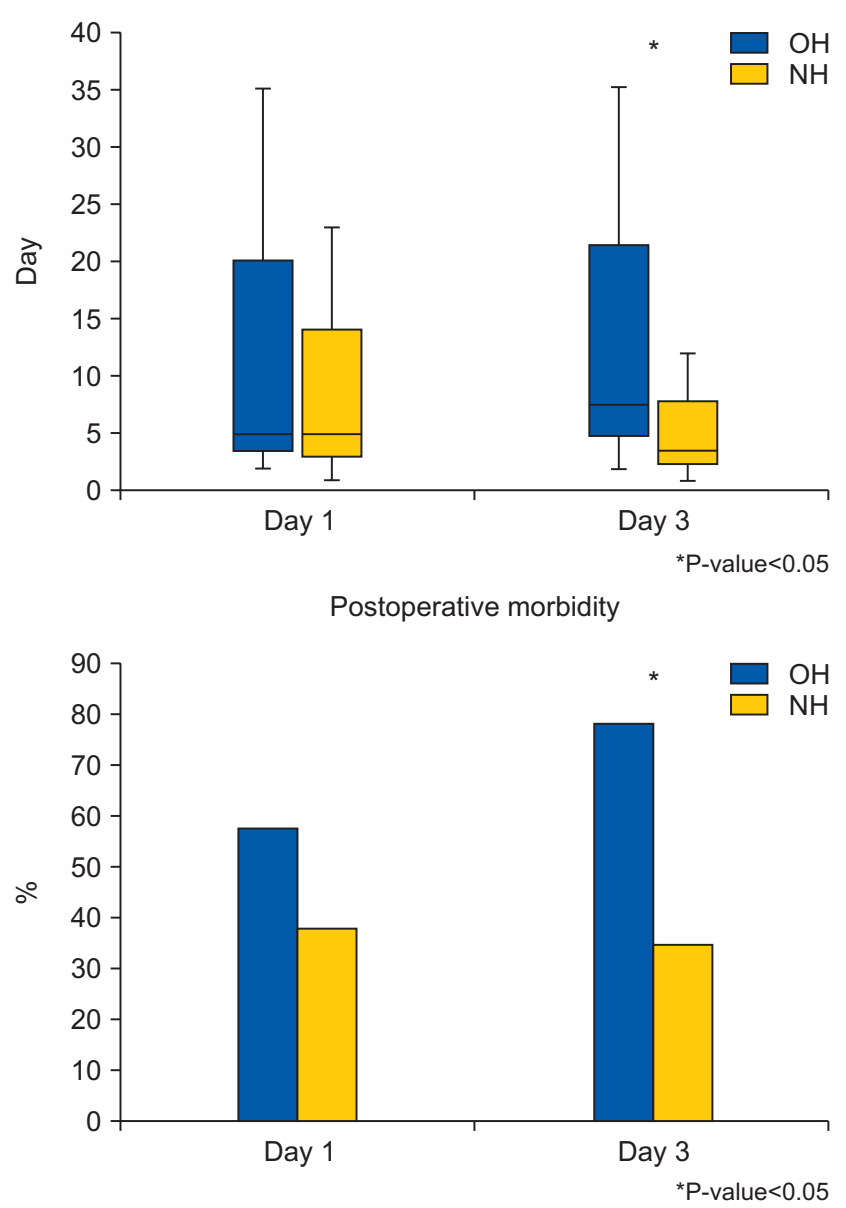

Length of hospital stay
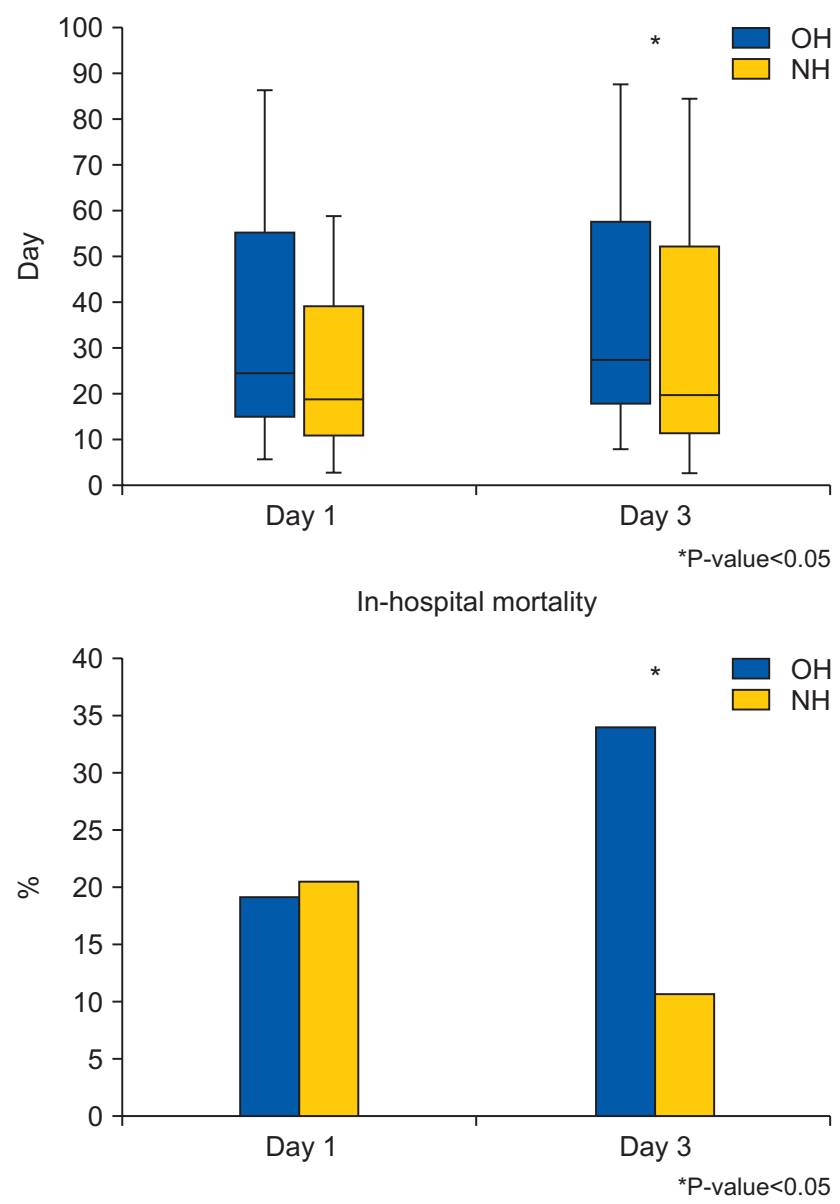

Fig. 2. Differences in clinical outcomes between overhydration group $(\mathrm{OH})$ and normohydration group (NH) during 3 days after surgery. 
Table 3. Multivariate analyses of independent risk factors for the occurrence of (A) postoperative morbidities* and (B) in-hospital mortality

(A) Postoperative morbidities

\begin{tabular}{lccr}
\multicolumn{1}{c}{ Variable } & Odds ratio & 95\% CI & P-value \\
\hline Age & 1.015 & $0.983 \sim 1.048$ & 0.364 \\
Shock on ICU admission & 1.274 & $0.266 \sim 6.114$ & 0.762 \\
ECW ratio at Day 1 & $9.79^{*} 10^{3}$ & $0.000 \sim 2.778^{*} 10^{15}$ & 0.495 \\
SOFA score at ICU admission & 1.176 & $0.966 \sim 1.431$ & 0.107 \\
Overhydration at Day 3 & 5.709 & $2.199 \sim 14.819$ & $<0.001$ \\
\hline
\end{tabular}

*Postoperative morbidities were defined as the occurrence of morbidities as grade 3 or more according to the Clavien-Dindo classification.[6]

(B) In-hospital mortality

\begin{tabular}{lclc}
\hline \multicolumn{1}{c}{ Variable } & Odds ratio & \multicolumn{1}{c}{ 95\% CI } & P-value \\
\hline Shock on ICU admission & 2.079 & $0.477 \sim 9.060$ & 0.330 \\
ECW ratio at Day 1 & 1.200 & $0.000 \sim 1.35^{*} 10^{5}$ & 0.247 \\
SOFA score at ICU admission & 1.132 & $0.948 \sim 1.353$ & 0.171 \\
Overhydration** at Day 3 & 4.244 & $1.398 \sim 12.883$ & 0.045 \\
\hline
\end{tabular}

${ }^{* *}$ Overhydration is defined as the case where the value of ECW ratio (ECW/TBW) is above 0.390.[7-9]

surgery due to massive transfusion and applying fluid as the means of resuscitation to correct the loss of body fluid. The fluctuation and imbalance of volume status are most remarkable in the acute phase of postoperative patient that is expected to involve higher loss of blood volume in vessels and the higher requirement to replenish it. As observed in our study in Fig. 1, this phenomenon is more pronounced in patients undergoing abdominal surgery compared to endovascular surgery that is performed without massive tissue injury. It is because that the change in volume status after endovascular surgery is relatively small and also the need for massive fluid resuscitation is less than that of abdominal surgery due to the low need of dietary restrictions after surgery. Therefore, in the case of critically ill patients who had undergone abdominal surgery as in this study, most cases require aggressive fluid management. However, an excessive effort of readjusting the fluid imbalance and replenishing it can result in the shift of fluid into the extracellular space through the injured tissue or vessel during the surgery, that could in turn cause respiratory complications such as pulmonary edema. [10] This can also lead to the decline of tissue oxygenation and cause tissue edema, which could in turn culminate in cell necrosis, the possible cause of the delay in wound healing or anastomosis leakage. On the contrary, the insufficient replacement of fluid in the acute phase could cause several problems as the insufficient in tissue perfusion and organ impairments including AKI, hepatic dysfunction, and decreased bowel motility. Thus, it would be important to maintain the balance in a patient's volume status by properly assessing the amount of fluid needed in order to improve the prognosis.[11]

Actually, a very acute postoperative phase, as the first 24 hours right after major surgery, is the period where the loss of interstitial or intravascular fluid is common due to the direct consequences of the surgery, such as major tissue injuries or damages of vascular wall structures. Therefore, an appropriate tissue perfusion through fluid resuscitation is very important during this period, and the positive fluid imbalance in this period would be considered the natural course, not relevant to poor prognosis.[12,13] Rather, it would be more important to examine a patient's volume status and prevent problems resulted from inappropriate fluid management in the acute phase, 48 to 72 hours after major abdomen surgery. In acute phase, the previous oliguria caused by the secretion of the stress hormone and the increase in renal reabsorption due to tissue injury and volume loss, would be usually improved and then progresses to the diuretic phase. As reported in previous studies of Boerma et al. [14] and Cordemans et al. $[10,15]$, on the third day of shock, microcirculatory blood flow begins to back to normal and the homeostasis of cytokines in healing microcirculatory disruptions begins to work, that lead to the closure of the capillary leak. Therefore, we suppose that the positive fluid imbalance observed in the period of 48 to 72 hours after the surgery would be related to organ dysfunctions and thus relevant to the prognosis of disease.

In this study, the ECW ratio of the postoperative patient turned out to be highest value on the second day of ICU admission, and then it tended to decrease. The ECW ratio is known to reflect edematous status and malnutrition in patients and we found that a significant difference in the outcomes between groups classified by the ECW ratio. That implies the usefulness of ECW ratio as the assessment tool of the volume status in acute phase after abdomen surgery. As shown in the result of multivariate analysis, the overhydration 
status defined by ECW ratio on the third day of ICU would be the significant factors of predicting in postoperative complications and in-hospital mortality, respectively. This supports that a proper volume status management in the acute phase, 48 to 72 hours after the surgery would have clinical importance.

Also, BIA can provide an assumption on a patient's body composition and volume status and quantifies the amounts of extracellular and intracellular water of the patient's body. $[1,16]$ The fact that the measurement using BIA usually takes less than two minutes and it is non-invasive with a portable machine suitable for an immobilized patient. It can be used safely for the postoperative critical ill patients in ICU in a poor clinical condition concerned with bleeding or fluid loss during or immediately after surgery without time-related discomfort or any other procedure-related risks. In fact, there were no complication as relevant to the BIA use in our study. Thus, BIA could be helpful for measuring the volume status of critical ill patients, especially to those in acute phase who suffer from severe pain due to operative wounds or multiple drains, that is too serious to take repeated invasive examinations.

Despite of interesting outcomes, the results in current study should be interpreted with caution due to various limitations. Firstly, our data had some bias of analysis due to its observational studied design of single center. To compensate this limitation, we strictly enrolled participants according to inclusion criteria and dropped out according to the exclusion criteria. Next, various factors which could interfere with BIA measurement and affect BIA values such as the infusions with large amounts of fluids, peripheral edema was not fully understood. The measurement of body weight could be inaccurate because of the multiple drainage, fluid lines, devices, and even heavy bedclothes. Various factors of ICU environment such as ambient air, skin temperature, seating or fever of patient, or specific conductance of ICU bed are also not understood about its influence on the BIA measurement. The insufficient data about insensible fluid loss due to absence of indirect calorimetry in our institution might be another factor of this bias. Thirdly, we compared and analyzed the values at a particular day and time in the morning to measure the BIA parameters and fluid balance analysis. Therefore, it is difficult to rule out the possibility that the effect of the fluid balance before the day of measurement increased or overlaid. As mentioned in the manuscript, we believe that the fluid balance was relatively constant before the third day of post-operation for the patients in this study who were subject to general cases, except for the extraordinary cases that involved patients who died within two days after the operation, thus we expect that the cumulative effect and bias may be relatively small. Even though out suppositions, the further study in near future should be complemented to analyze the changes in BIA parameters caused by the daily differences of fluid status. Additionally, although the SOFA score and op- eration time during postoperative day showed no statistically significant difference between the overhyration group and normohydration group in our results, the patient's underlying disease or the disease severity can affect the cellular function during the recovery process. Thus, it can also act as the factors that influence the volume status. We supposed that the future study may show the possibility of identifying the effect of these factors when being conducted with a larger number of patients. Lastly, we did not perform the subgroup analysis according to the type of shock or type of surgery such as open or laparoscopic surgery. The subgroup analysis depending on the sort of operations or intervention procedure should be performed in near future to develop a detailed guideline.

Nevertheless, the current study will be meaningful in that it differs from previous studies because this is a prospective cohort study conducted on patients after major abdomen surgery. Also, this study enrollment was strictly limited to ICU patients in the acute phase. These patients usually show the most physiologically dynamic changes with unstable vital signs during this period, thus they generally require large amounts of fluids and are vulnerable to complications caused by inappropriate volume status. We believe that BIA can provide a foundation of the fluid management guideline in this early phase of critical ill patients after major operations.

In conclusion, the use of BIA to monitor volume status is a feasible method that is easy, safe, and adaptable for critical patients in immobilization status after major abdomen surgery. The overhydration status on Day 3 after surgery, was related to postoperative morbidity and in-hospital mortality occurrence. Based on this, it is expected that for postoperative patients in the acute phase who receive intensive care, monitoring through BIA parameters within $48 \sim 72 \mathrm{hrs}$ can improve the prognosis, consecutively.

\section{STATEMENT OF INFORMED CONSENT}

This study was approved and carefully monitored by our Institutional Review Board. (No. IRB; KC19RESI0584) Patients provided written informed consent from each participant, and the procedures were in compliance with Helsinki Declaration.

\section{AUTHORS' CONTRIBUTIONS}

Study conception and design: EYK. Acquisition of data: YJC, EYK. Statistical analysis: YJC, EYK. Analysis and interpretation of data: YJC, EYK. Drafting of manuscript: YJC. Critical revision: YJC, EYK. Guarantor of article: EYK. All authors approved the final version of the article, including the authorship list. 


\section{CONFLICTS OF INTEREST}

No potential conflict of interest relevant to this article was reported.

\section{ORCID}

Yoon Ji Chung, https://orcid.org/0000-0001-6194-7749

Eun Young Kim, https://orcid.org/0000-0003-4408-4165

\section{REFERENCES}

1. Lee YH, Lee JD, Kang DR, Hong J, Lee JM. Bioelectrical impedance analysis values as markers to predict severity in critically ill patients. J Crit Care 2017;40:103-7.

2. Yilmaz Z, Yildirım Y, Aydin FY, Aydin E, Kadiroğlu AK, Yilmaz $M E$, et al. Evaluation of fluid status related parameters in hemodialysis and peritoneal dialysis patients: clinical usefulness of bioimpedance analysis. Medicina (Kaunas) 2014;50:269-74.

3. Basso F, Berdin G, Virzì GM, Mason G, Piccinni P, Day S, et al. Fluid management in the intensive care unit: bioelectrical impedance vector analysis as a tool to assess hydration status and optimal fluid balance in critically ill patients. Blood Purif 2013;36:192-9.

4. Barbosa-Silva MC, Barros AJ. Bioelectric impedance and individual characteristics as prognostic factors for postoperative complications. Clin Nutr 2005;24:830-8.

5. Lukaski HC, Kyle UG, Kondrup J. Assessment of adult malnutrition and prognosis with bioelectrical impedance analysis: phase angle and impedance ratio. Curr Opin Clin Nutr Metab Care 2017;20:330-9.

6. Dindo D, Demartines N, Clavien PA. Classification of surgical complications: a new proposal with evaluation in a cohort of 6336 patients and results of a survey. Ann Surg 2004;240:205-13.

7. Noda Y, Suzuki H, Kanai T, Samejima Y, Nasu S, Tanaka A, et al. The association between extracellular water-to-total body water ratio and therapeutic durability for advanced lung cancer. Anticancer Res 2020;40:3931-7.
8. Nishikawa H, Yoh K, Enomoto H, Ishii N, Iwata Y, Nakano $\mathrm{C}$, et al. Extracellular water to total body water ratio in viral liver diseases: a study using bioimpedance analysis. Nutrients 2018;10:1072.

9. Park $\mathrm{KH}$, Shin JH, Hwang JH, Kim SH. Utility of volume assessment using bioelectrical impedance analysis in critically ill patients receiving continuous renal replacement therapy: a prospective observational study. Korean J Crit Care Med 2017;32:256-64.

10. Cordemans C, De Laet I, Van Regenmortel N, Schoonheydt K, Dits H, Huber W, et al. Fluid management in critically ill patients: the role of extravascular lung water, abdominal hypertension, capillary leak, and fluid balance. Ann Intensive Care 2012;2(Suppl 1 Diagnosis and management of intra-abdominal hyperten):S1.

11. Kayilioglu SI, Dinc T, Sozen I, Bostanoglu A, Cete M, Coskun F. Postoperative fluid management. World J Crit Care Med 2015;4:192-201.

12. Malbrain MLNG, Van Regenmortel N, Saugel B, De Tavernier B, Van Gaal PJ, Joannes-Boyau O, et al. Principles of fluid management and stewardship in septic shock: it is time to consider the four D's and the four phases of fluid therapy. Ann Intensive Care 2018;8:66.

13. Silversides JA, Major E, Ferguson AJ, Mann EE, McAuley DF, Marshall JC, et al. Conservative fluid management or deresuscitation for patients with sepsis or acute respiratory distress syndrome following the resuscitation phase of critical illness: a systematic review and meta-analysis. Intensive Care Med 2017;43:155-70.

14. Boerma EC, van der Voort PH, Spronk PE, Ince C. Relationship between sublingual and intestinal microcirculatory perfusion in patients with abdominal sepsis. Crit Care Med 2007;35:1055-60.

15. Rivers EP. Fluid-management strategies in acute lung injury--liberal, conservative, or both? N Engl J Med 2006; 354:2598-600

16. Malbrain ML, Huygh J, Dabrowski W, De Waele JJ, Staelens A, Wauters J. The use of bio-electrical impedance analysis (BIA) to guide fluid management, resuscitation and deresuscitation in critically ill patients: a bench-to-bedside review. Anaesthesiol Intensive Ther 2014;46:381-91. 